\title{
The Relationship Between Severe Complications, Beta-Blocker Therapy and Long-Term Survival Following Emergency Surgery for Colon Cancer
}

\author{
Rebecka Ahl ${ }^{1,2} \cdot$ Peter Matthiessen ${ }^{2,3} \cdot$ Yang Cao $^{4} \cdot$ Gabriel Sjolin $^{2,5} \cdot$ Olle Ljungqvist $^{6,7}$ • \\ Shahin Mohseni ${ }^{2,8}$
}

\begin{abstract}
Background Emergency surgery for colon cancer carries significant morbidity, and studies show more than doubled mortality when comparing elective to emergency surgery. The relationship between postoperative complications and survival has been outlined. Beta-blocker therapy has been linked to improved postoperative outcomes. This study aims to assess the impact of postoperative complications on long-term survival following emergency surgery for colon cancer and to determine whether beta-blockade can reduce complications.

Study design This cohort study utilized the prospective Swedish Colorectal Cancer Registry to identify adults undergoing emergency colon cancer surgery between 2011 and 2016. Prescription data for preoperative beta-blocker therapy were collected from the national drug registry. Cox regression was used to evaluate the effect of beta-blocker exposure and complications on 1-year mortality, and Poisson regression was used to evaluate beta-blocker exposure in patients with major complications.

Results A total of 3139 patients were included with a mean age of 73.1 [12.4] of which 671 (21.4\%) were prescribed beta-blockers prior to surgery. Major complications occurred in $375(11.9 \%)$ patients. Those suffering major complications showed a threefold increase in 1-year mortality (adjusted HR $=3.29 ; 95 \%$ CI $2.75-3.94 ; p<0.001$ ). Betablocker use was linked to a $60 \%$ risk reduction in 1-year mortality (adjusted HR $=0.40$; 95\% CI 0.26-0.62; $p<0.001$ ) but did not show a statistically significant association with reductions in major complications (adjusted IRR $=0.77 ; 95 \%$ CI $0.59-1.00 ; p=0.055$ ).

Conclusion The development of major complications after emergency colon cancer surgery is associated with increased mortality during one year after surgery. Beta-blocker therapy may protect against postoperative complications.
\end{abstract}

Shahin Mohseni

shahin.mohseni@regionorebrolan.se

Rebecka Ahl

rebecka.ahl@sll.se

Peter Matthiessen

peter.matthiessen@ regionorebrolan.se

Yang Cao

yang.cao@oru.se

Gabriel Sjolin

gabriel.sjolin@regionorebrolan.se
Olle Ljungqvist

olle.ljungqvist@oru.se

1 Department of Surgery, Karolinska University Hospital, Stockholm, Sweden

2 School of Medical Sciences, Örebro University, Örebro, Sweden

3 Division of Colorectal Surgery, Department of Surgery, Örebro University Hospital, Örebro, Sweden 


\section{Background}

Postoperative complications after major surgery have been associated with decreased short- and long-term survival $[1,2]$. Emergency laparotomy is in itself considered a highrisk procedure with considerable postoperative morbidity and mortality rates. Clarke et al. outlined a 30-day mortality rate of just under $20 \%$ following emergency laparotomy. This number was doubled when considering patients over the age of 80 . They also found a need for intensive care interventions in every fourth patient undergoing emergency laparotomy [3]. The presence of a postoperative complication appears more important than high preoperative patient risk factors in determining survival following major surgery. Khuri et al. found that the occurrence of a complication in the 30-day postoperative period reduced median patient survival by 69\% [2]. Despite advances in surgical techniques as well as pre- and postoperative care, a high incidence of complications and early mortality remain following emergency abdominal surgery for colon cancer $[4,5]$. Sjo et al. showed that mortality increased from 3.5 to $10 \%$ and complications from 24 to $38 \%$ when elective surgery is compared to emergency surgery for colon cancer [5]. There is, however, a paucity of data in the literature on the long-term effects of postoperative complications after emergency surgery for colon cancer and the factors which may reduce them.

Beta-blocker exposure has demonstrated a protective effect with decreased 30-day mortality following emergency surgery for colon cancer [6]. In surgery for rectal cancer, preoperative beta-blocker therapy has been associated with a decrease in several major postoperative complications and an increase in survival rate up to 1-year postoperatively [7]. It has been suggested that these positive effects are due to the down-regulation of the deleterious effects of the hyperadrenergic state induced by major surgery [8]. The hypothesis of the current study is that major postoperative complications are associated with worse long-term survival after emergency surgery for colon cancer, and that beta-blocker therapy may be used to

4 Clinical Epidemiology and Biostatistics, School of Medical Sciences, Örebro University, Örebro, Sweden

5 Department of Surgery, Örebro University Hospital, Örebro, Sweden

6 Department of Surgery, School of Medical Sciences, Faculty of Medicine and Health, Örebro University, Örebro, Sweden

7 Department of Molecular Medicine and Surgery, Karolinska Institute, Stockholm, Sweden

8 Division of Trauma and Emergency Surgery, Department of Surgery, Örebro University Hospital, Örebro, Sweden decrease the incidence of major postoperative complications and thereby improve survival.

\section{Materials and methods}

\section{Study population}

Ethical approval was obtained from the Institutional Review Board of Uppsala County, Sweden (Ref. 2015/454). Subsequently, all adults aged $\geq 18$ years who underwent emergency surgery for colon cancer between January 1, 2011, and October 31, 2016, were identified from the Swedish Colorectal Cancer Registry (SCRCR). The SCRCR is a nationwide registry with prospectively collected data. The registry is used for quality measurements and has a completeness of over 99\% [9]. Patients undergoing laparotomy without bowel resection were excluded from the cohort. Variables accrued for each patient included age, body mass index (BMI), sex, complications classified according to Clavien-Dindo, tumor location, TNM classification, American Society of Anesthesiologists (ASA) classification, indication for surgery and type of surgery. Data on 1-year mortality were retrieved from the Swedish Cause of Death Registry maintained by the National Board of Health and Welfare. Complications with a Clavien-Dindo score of three or greater were classified as major. This definition was used since the nature of the surgery coupled to patient and disease characteristics in the current study is inevitably associated with higher rates of minor complications.

The primary aim of the study was to assess the association of major postoperative complications with 1-year mortality. The secondary aims were to assess the interaction between beta-blocker use and the incidence of major postoperative complications, as well as the effect of betablocker exposure on mortality in patients suffering a major postoperative complication.

\section{Beta-blocker therapy}

Prescription information with regards to beta-blocker therapy was sought from the Swedish national drug registry of the National Board of Health and Welfare. Unique personal identity numbers were used to link study subjects to the national database for drug prescriptions. All drug prescriptions in Sweden are registered in the national database including drug type, dosage and date of issue and date of collection. All patients with a prescription prior to the date of surgery were allocated to the beta-blocker positive group. The most recently collected beta-blocker prescription was used to reflect type and dosage. Collated drugs information included drug name, dosage, frequency 
of administration (daily or as required), prescription date of issue and collection. Only patients with collected prescriptions for daily beta-blocker administration, as opposed to 'use when required,' met inclusion criteria for betablocker positive cases. Patients were divided into betablocker positive and negative groups as well as a major postoperative complication group and non-major postoperative complication group for analysis.

\section{Statistical analysis}

Patient demographics, clinical information and outcomes were compared between the group with major complications and the rest of the study population. The Chi-squared test or Fisher's exact test was used for categorical variables. Student's $t$ test was used for normally distributed continuous variables and the Mann-Whitney U test was used for ordinal or asymmetrically distributed continuous variables.

The proportional hazards Cox regression model was used to evaluate the effect of complications and betablocker exposure on mortality, and the interaction between complications and beta-blocker therapy. The event was defined as death of any cause within one year of surgery, and the censoring was defined as alive at one year. The Poisson regression model was used to evaluate the effect of beta-blocker use on severe complications. The HuberWhite sandwich estimator for variance was used in parameter estimation, which is robust to some types of model misspecifications as long as the observations are independent. To adjust for potential confounding factors, the following covariates were included in the regression models: age, gender, BMI, ASA, TNM classification, cancer stage, type of surgical resection and the indication for emergency surgery. Multiple imputation method was used for handling missing values in BMI and ASA scores by using the iterative Markov chain Monte Carlo method [10]. Five imputed data sets were generated and the estimates from the five data sets were combined according to Rubin's rules [11]. The results of the above outlined risk assessments are reported as hazard ratio (HR) for 1-year mortality or as incidence rate ratio (IRR) with corresponding 95\% confidence interval (CI). Results were considered statistically significant at a two-sided p-value of less than 0.05. Statistical analyses were conducted in Stata 15.1 (StataCorp, College Station, TX, USA).

\section{Results}

A total of 3139 patients met inclusion criteria. The mean age (standard deviation) of the total studied cohort was 73.1 (12.4) years and $1666(53.1 \%)$ were women. The majority of patients had stage III cancer $(41.9 \%)$ with the most common indication for emergency surgery being bowel obstruction (78.1\%). Six hundred and seventy-one (21.4\%) patients were on regular beta-blocker therapy at time of surgery (Table 1). The most commonly prescribed beta-blockers were: metoprolol $(57.5 \%)$, followed by bisoprolol (20.4\%) and atenolol (10.7\%), with the remaining percentage $(11.3 \%)$ being made up of a mixture. The majority of patients on metoprolol had a dose of $50 \mathrm{mg}$ (50\%), followed by $100 \mathrm{mg}(25 \%)$ and $25 \mathrm{mg}(25 \%)$. For bisoprolol, the dosages were $10 \mathrm{mg}(20 \%), 5 \mathrm{mg}(50 \%)$ and $2.5 \mathrm{mg}(30 \%)$. For atenolol, $50 \%$ were on a dose of $50 \mathrm{mg}$ and the remainder had a dose of $25 \mathrm{mg}$. The mean percentage of adults prescribed beta-blockers in the general population of Sweden during the study period was $13.1 \%$. This percentage increased to $29.4 \%$ for the age group 60 to 85 years (information collected from the national drug registry of the National Board of Health and Welfare, October 1, 2018).

Major complications occurred in 375 (11.9\%) patients. Complications consisted of cardiovascular events $(28.5 \%)$, anastomotic dehiscence (24.8\%), intra-abdominal abscess formation $(17.9 \%)$, stoma-related complications (16\%), sepsis $(15.5 \%)$, wound infection $(9.1 \%)$, pneumonia $(9.1 \%)$, bleeding $(5.1 \%)$, cerebrovascular insults (CVI) $(2.9 \%)$ and deep vein thrombosis (DVT) (1.1\%). Unspecified complications were noted in $3.2 \%$ of the cohort. Patients suffering severe complications were older (75.3 vs. 72.8 years, $p<0.001$ ), less fit for surgery (ASA $\geq 3$ in $61.3 \%$ vs. $44.5 \%, p<0.001)$ and more commonly had bowel perforation as the surgical indication (25.6\% vs. $15.6 \%, p<0.001$ ) (Table 1). There were no statistical differences in sex, surgical procedure, overall cancer stage or exposure to beta-blockers between patients who did and did not suffer a major postoperative complication (Table 1).

Patients suffering major complications demonstrated a higher crude 1 -year mortality rate $(53.6 \%$ vs $23.1 \%$, $p<0.001$ ) (Table 1). Cox regression analysis and complementary Kaplan-Meier curves outlined a threefold increase in 1-year mortality risk of this subgroup (adjusted $\mathrm{HR}=3.29 ; \quad 95 \%$ CI 2.75-3.94; $p<0.001$ ) (Table 2, Fig. 1left). This relationship remained after excluding patients who died within 30-days of surgery to control for early postoperative deaths (adjusted HR $=1.87$; 95\% CI 1.44-2.44; $p<0.001$ ) (Fig. 1 right). In all patients, betablocker therapy was associated with a $34 \%$ risk reduction in overall 1-year mortality (adjusted HR $=0.66$; 95\% CI $0.53-0.82 ; p<0.001$ ) (Table 2). The most common cause of death up to 1-year postoperatively was multi-organ failure $(47.6 \%)$, followed by cardiovascular $(16.7 \%)$, pulmonary $(15.0 \%)$, sepsis $(12.3 \%)$ and cerebrovascular $(5.3 \%)$. The cause of death was unknown in $3.1 \%$ of 
Table 1 Patient demographics and clinical outcomes for total cohort and subdivided according to complication severity, major complication being Clavien-Dindo $\geq 3$

\begin{tabular}{|c|c|c|c|c|}
\hline & $\begin{array}{l}\text { Total } \\
n=3139\end{array}$ & $\begin{array}{l}\text { No major postoperative } \\
\text { complication } \\
n=2764\end{array}$ & $\begin{array}{l}\text { Major postoperative } \\
\text { complication } \\
n=375\end{array}$ & $p$ \\
\hline 1-year mortality, $n(\%)$ & $840(26.8)$ & $639(23.1)$ & $201(53.6)$ & $<0.001$ \\
\hline Beta-blocker use, $n(\%)$ & $671(21.4)$ & $598(21.6)$ & $73(19.5)$ & 0.37 \\
\hline Sex, $n(\%)$ & & & & 0.41 \\
\hline Male & $1473(46.9)$ & 1289 (46.6) & $184(49.1)$ & \\
\hline Female & $1666(53.1)$ & $1475(53.4)$ & $191(50.9)$ & \\
\hline Age in years, mean (SD) & $73.1(12.4)$ & $72.8(12.4)$ & $75.3(12.1)$ & $<0.001$ \\
\hline BMI, mean (SD) & $25.1(4.5)$ & $25.1(4.5)$ & $25.2(4.8)$ & 0.74 \\
\hline ASA classification, $n(\%)$ & & & & $<0.001$ \\
\hline 1 & $305(9.7)$ & $287(10.4)$ & $18(4.8)$ & \\
\hline 2 & $1281(40.8)$ & $1168(42.3)$ & $113(30.1)$ & \\
\hline 3 & $1226(39.1)$ & $1059(38.3)$ & $167(44.5)$ & \\
\hline 4 & $226(7.2)$ & $166(6.0)$ & $60(16.0)$ & \\
\hline 5 & $8(0.3)$ & $5(0.2)$ & $3(0.8)$ & \\
\hline Missing & $93(3.0)$ & $79(2.9)$ & $14(3.7)$ & \\
\hline ASA, median (IQR) & $2[2,3]$ & $2[2,3]$ & $3[2,3]$ & $<0.001$ \\
\hline Surgical procedure, $n(\%)$ & & & & 0.36 \\
\hline Ileocecal resection & $125(4.0)$ & 107 (3.9) & $18(4.8)$ & \\
\hline Right-sided hemicolectomy & $1467(46.7)$ & $1302(47.1)$ & $165(44.0)$ & \\
\hline Resection of transverse colon & $53(1.7)$ & $42(1.5)$ & $11(2.9)$ & \\
\hline Left-sided hemicolectomy & $397(12.6)$ & $354(12.8)$ & $43(11.5)$ & \\
\hline Sigmoid resection & $451(14.4)$ & $397(14.4)$ & $54(14.4)$ & \\
\hline Total colectomy & $229(7.3)$ & $199(7.2)$ & $30(8.0)$ & \\
\hline Anterior resection & $42(1.3)$ & $40(1.4)$ & $2(0.5)$ & \\
\hline Hartmann's procedure & $325(10.4)$ & $279(10.1)$ & $46(12.3)$ & \\
\hline Other operation & $50(1.6)$ & $44(1.6)$ & $6(1.6)$ & \\
\hline \multicolumn{5}{|l|}{ Surgery indication, $n(\%)$} \\
\hline Obstruction & $2450(78.1)$ & $2181(78.9)$ & 269 (71.7) & 0.002 \\
\hline Bleeding & $90(2.9)$ & $80(2.9)$ & $10(2.7)$ & 0.93 \\
\hline Bowel perforation & $526(16.8)$ & $430(15.6)$ & $96(25.6)$ & $<0.001$ \\
\hline Other & $236(7.5)$ & $202(7.3)$ & $34(9.1)$ & 0.27 \\
\hline T Stage, $n(\%)$ & & & & 0.06 \\
\hline $1-2$ & $132(4.2)$ & $123(4.5)$ & $9(2.4)$ & \\
\hline 3 & $1590(50.7)$ & $1410(51.0)$ & $180(48.0)$ & \\
\hline 4 & $1405(44.8)$ & $1222(44.2)$ & $183(48.8)$ & \\
\hline Missing & $12(0.4)$ & $9(0.3)$ & $3(0.8)$ & \\
\hline $\mathrm{N}$ stage, $n(\%)$ & & & & 0.10 \\
\hline 0 & $1143(36.4)$ & $1011(36.6)$ & $132(35.2)$ & \\
\hline N 1-2 & $1983(63.2)$ & $1744(63.1)$ & $239(63.7)$ & \\
\hline Missing & $13(0.4)$ & $9(0.3)$ & $4(1.1)$ & \\
\hline M stage, $n(\%)$ & & & & 0.76 \\
\hline 0 & $2361(75.2)$ & $2076(75.1)$ & $285(76.0)$ & \\
\hline 1 & $778(24.8)$ & $688(24.9)$ & $90(24.0)$ & \\
\hline Cancer stage $n(\%)$ & & & & 0.34 \\
\hline 1 & $95(3.0)$ & $89(3.2)$ & $6(1.6)$ & \\
\hline 2 & $951(30.3)$ & $836(30.2)$ & $115(30.7)$ & \\
\hline
\end{tabular}


Table 1 continued

\begin{tabular}{|c|c|c|c|c|}
\hline & $\begin{array}{l}\text { Total } \\
n=3139\end{array}$ & $\begin{array}{l}\text { No major postoperative } \\
\text { complication } \\
n=2764\end{array}$ & $\begin{array}{l}\text { Major postoperative } \\
\text { complication } \\
n=375\end{array}$ & $p$ \\
\hline 3 & $1315(41.9)$ & 1151 (41.6) & $164(43.7)$ & \\
\hline 4 & $778(24.8)$ & $688(24.9)$ & $90(24.0)$ & \\
\hline \multicolumn{5}{|c|}{ Major complications (Clavien-Dindo $\geq 3) n(\%)$} \\
\hline Sepsis & & & $58(15.5)$ & \\
\hline Pneumonia & & & $34(9.1)$ & \\
\hline Anastomotic dehiscence & & & $93(24.8)$ & \\
\hline DVT & & & $4(1.1)$ & \\
\hline CVI & & & $11(2.9)$ & \\
\hline Wound infection & & & $34(9.1)$ & \\
\hline Intra-abdominal abscess & & & $67(17.9)$ & \\
\hline Bleeding & & & $19(5.1)$ & \\
\hline Stoma-related & & & $16(4.3)$ & \\
\hline Cardiovascular & & & $107(28.5)$ & \\
\hline Other & & & $12(3.2)$ & \\
\hline
\end{tabular}

$B M I$ body mass index, ASA American Society of Anesthesiologists, DVT deep vein thrombosis, $C V I$ cerebrovascular insult

patients. An interaction between beta-blocker exposure and major postoperative complications was noted with a hazard ratio of 0.65 (95\% CI $0.40-1.03$ ) indicating a further $35 \%$ risk reduction in 1-year mortality for patients with a major complication and on beta-blocker therapy. This interaction was, however, not statistically significant with a $p$ value of 0.067 (Table 2). Notably, patients with major complications had almost a three times higher mortality rate compared to those not suffering a major complication (Table 1).

A trend toward decreased overall incidence of major postoperative complications was observed in the betablocker positive patients (adjusted IRR 0.77, 95\% CI $0.59-1.00, p=0.055$ ) (Table 3). In the event that a major postoperative complication occurred, beta-blocker exposure significantly reduced the risk of mortality with $60 \%$ $(\mathrm{HR}=0.40 ; 95 \%$ CI $0.26-0.62 ; p<0.001)$ in the first postoperative year (Table 4). For specific complications, there was a significant reduction in 1-year mortality seen in patients suffering from postoperative sepsis $(\mathrm{HR}=0.09$; $95 \%$ CI $0.02-0.37 ; p=0.001)$, cardiovascular event $(\mathrm{HR}=0.33 ; 95 \%$ CI $0.17-0.63 ; p=0.001)$ and CVI $(\mathrm{HR}=0.03 ; 95 \%$ CI $0.002-0.54 ; p=0.017)$ (Table 4).

\section{Discussion}

The current study shows that major postoperative complications after emergency laparotomy and resection surgery for colon cancer are associated with a significantly raised mortality during the first postoperative year. Patients on regular preoperative beta-blocker therapy showed an almost significant trend toward reduced risk of developing several major postoperative complications. More interestingly, patients on beta-blocker therapy who developed severe sepsis, a severe cardiovascular event or cerebrovascular insult demonstrated significantly better survival than those not receiving such therapy with same adverse postoperative events. To the authors best knowledge, this is the first study investigating the impact of major complications on long-term survival following surgery for emergency colon cancer, the association between beta-blocker therapy and the incidence of such adverse events, and the effect of beta-blocker therapy on long-term mortality in patients who develop major postoperative complications.

Emergency laparotomy is associated with high morbidity and mortality [3]. When it comes to emergency laparotomy with tumor resection for colon cancer, data from the American College of surgeons' National Quality Improvement Program have demonstrated a higher incidence of serious postoperative complications and mortality compared to equivalent elective surgical procedures [4]. Similar findings have been outlined in other studies comparing elective to emergency surgery in the context of colon cancer [5, 12]. There is mounting evidence, suggesting that postoperative complications after major surgery have long-term negative effects on survival $[1,2]$. Results reported in a prospective multicenter study including over 100,000 patients from the American College of Surgeon's National Surgical Quality Improvement subjected to eight different major surgical procedures, found a 
Table 2 Hazard ratios for risk factors for 1-year mortality

\begin{tabular}{|c|c|c|c|}
\hline Variable & Adjusted HR & $95 \% \mathrm{CI}$ & $p$ \\
\hline Major postoperative complication (Clavien-Dindo $\geq 3$ ) & 3.29 & $2.75-3.93$ & $<0.001$ \\
\hline Preoperative BB therapy & 0.66 & $0.53-0.80$ & $<0.001$ \\
\hline Severe complication $\times$ preoperative BB & 0.65 & $0.40-1.03$ & 0.067 \\
\hline Female sex & 1.15 & $0.99-1.32$ & 0.056 \\
\hline Age & 1.04 & $1.03-1.04$ & $<0.001$ \\
\hline BMI & 0.99 & $0.97-1.01$ & 0.786 \\
\hline \multicolumn{4}{|l|}{ ASA classification } \\
\hline 1 & Ref & & \\
\hline 2 & 1.40 & $0.95-2.06$ & 0.086 \\
\hline 3 & 2.52 & $1.71-3.70$ & $<0.001$ \\
\hline 4 & 4.69 & $3.09-7.12$ & $<0.001$ \\
\hline 5 & 18.65 & $8.03-43.28$ & $<0.001$ \\
\hline \multicolumn{4}{|l|}{ Surgical procedure } \\
\hline Ileocecal resection & Ref & & \\
\hline Right-sided hemicolectomy & 0.99 & $0.73-1.35$ & 0.994 \\
\hline Resection of transverse colon & 0.92 & $0.56-1.52$ & 0.755 \\
\hline Left-sided hemicolectomy & 0.61 & $0.41-0.90$ & 0.013 \\
\hline Sigmoid resection & 0.65 & $0.45-0.93$ & 0.020 \\
\hline Total colectomy & 0.89 & $0.61-1.30$ & 0.549 \\
\hline Anterior resection & 0.32 & $0.09-1.01$ & 0.054 \\
\hline Hartmann's & 0.65 & $0.45-0.94$ & 0.023 \\
\hline Other & 1.06 & $0.59-1.89$ & 0.833 \\
\hline \multicolumn{4}{|l|}{ Surgical indication } \\
\hline Obstruction & 1.32 & $1.01-1.71$ & 0.036 \\
\hline Bleeding & 1.88 & $1.26-2.79$ & 0.002 \\
\hline Bowel perforation & 1.85 & $1.44-2.36$ & $<0.001$ \\
\hline Other & 1.85 & $1.35-2.52$ & $<0.001$ \\
\hline \multicolumn{4}{|l|}{ T Stage } \\
\hline $1-2$ & Ref & & \\
\hline 3 & 0.64 & $0.30-1.32$ & 0.228 \\
\hline 4 & 0.89 & $0.43-1.85$ & 0.760 \\
\hline \multicolumn{4}{|l|}{$\mathrm{N}$ stage } \\
\hline 0 & Ref & & \\
\hline $1-2$ & 2.23 & $1.50-3.30$ & $<0.001$ \\
\hline \multicolumn{4}{|l|}{ M stage } \\
\hline 0 & Ref & & \\
\hline 1 & 3.72 & $1.38-9.98$ & 0.009 \\
\hline \multicolumn{4}{|l|}{ Cancer stage } \\
\hline 1 & Ref & - & \\
\hline 2 & 1.94 & $0.74-5.04$ & 0.173 \\
\hline 3 & 1.54 & $0.56-4.17$ & 0.400 \\
\hline 4 & 1.00 & (Omitted) & \\
\hline
\end{tabular}

$H R$ hazard ratio, $B B$ beta-blocker, $C I$ confidence interval, $B M I$ body mass index, $A S A$ American Society of Anesthesiologists

significant risk of long-term mortality (up to 8 years) in patients who developed a complication within 30-days postoperatively. Most notably, the occurrence of a postoperative complication was more important than preoperative patient risk profiles in determining long-term survival [2]. Similarly, Moonesinghe et al. demonstrated an independent relationship between postoperative morbidity and increased risk of death up to 3 years following major 

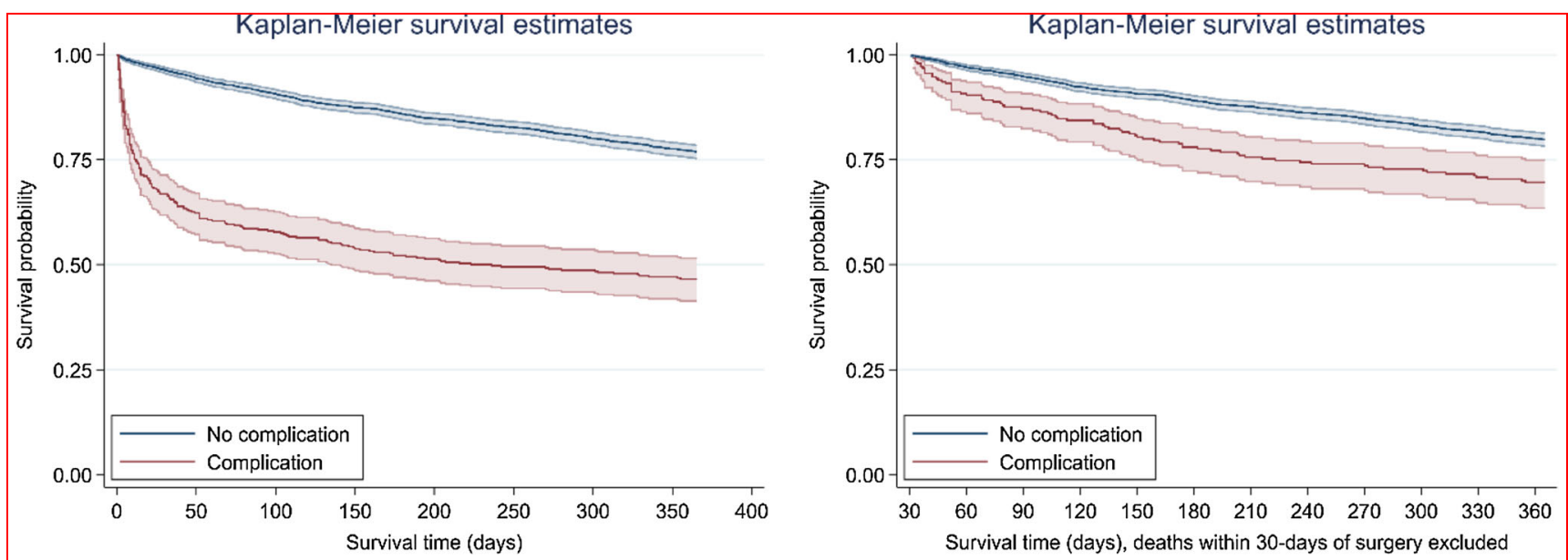

Fig. 1 Kaplan-Meier plot for 1-year survival (left) (HR = 3.29; 95\% CI 2.75-3.93; $p<0.001)$, and 1-year survival with deaths within 30-days of surgery excluded (right) $(\mathrm{HR}=1.87 ; 95 \%$ CI $1.44-2.44 ; p<0.001)$

non-cardiac, non-neuro elective surgery [1]. In the current study, a major postoperative complication, defined as a Clavien-Dindo $\geq 3$, was associated with a doubling of crude mortality ( 23.1 vs. $52.6 \%, p<0.001)$ and with a threefold higher risk of death up to one year after surgery (adjusted HR $=3.29$; 95\% CI 2.75-3.93).

It has been suggested that the hyperadrenergic state induced by major surgery or severe illness activates inflammatory responses leading to adverse outcomes

Table 3 Effects of beta-blocker exposure on major complications (Clavien-Dindo $\geq 3$ )

\begin{tabular}{llll}
\hline Complication & Adjusted IRR of BB & $95 \%$ CI & $\mathrm{p}$ \\
\hline Any major complication & 0.77 & $0.60-1.01$ & 0.055 \\
Sepsis & 0.80 & $0.42-1.51$ & 0.483 \\
Pneumonia* & 0.73 & $0.30-1.78$ & 0.488 \\
Anastomotic dehiscence & 0.71 & $0.40-1.24$ & 0.227 \\
DVT* $^{*}$ & 1.13 & $0.11-11.12$ & 0.918 \\
CVI** & 1.72 & $0.49-6.02$ & 0.395 \\
Wound infection** & 1.11 & $0.49-2.50$ & 0.811 \\
Intra-abdominal abscess & 1.08 & $0.59-1.95$ & 0.811 \\
Bleeding & 0.60 & $0.17-2.10$ & 0.426 \\
Stoma-related* & 0.26 & $0.03-1.96$ & 0.189 \\
Cardiovascular event & 0.93 & $0.59-1.47$ & 0.753 \\
Other & 0.66 & $0.14-3.17$ & 0.605 \\
\hline Pob & & &
\end{tabular}

Poisson regression model was used, adjusting for gender, age, BMI, ASA, surgical procedure, indication for surgery, TNM classification and overall cancer stage

$I R R$ incidence rate ratio, $B B$ beta-blockers, $C I$ confidence interval, $D V T$ deep vein thrombosis, $C V I$ cerebrovascular insult

*Adjusted for sex, age, BMI

**Adjusted for sex, age, BMI, ASA, surgical procedure
$[8,13]$. Beta-blocker administration has shown a downregulation of pro-inflammatory factors such as interleukin6 and TNF- $\alpha$ both in vivo and vitro which may offer some explanation to its beneficial use [14, 15]. Beta-blocker exposure reduced the incidence of several postoperative complications in the current study, but this finding did not reach statistical significance. One explanation for this could be due to the low number of occurred events of interest. Nevertheless, beta-blockers seem to offer a survival benefit in patients suffering a major postoperative complication. In patients with severe sepsis, beta-blocker exposure significantly reduced the mortality risk up to 1 -year postoperatively (adjusted HR $=0.09 ; 95 \%$ CI $0.02-0.37$ ). This is in line with previous findings by Khuri and colleagues, where a postoperative septic episode was associated with higher mortality rates up to 5 years after undergoing colectomy (63.2\% vs. 17.4\%) [2]. Furthermore, Fuchs et al. demonstrated that the continuation of a long-term beta-blocker in the acute phase of severe sepsis is associated with improved survival rates up to 90 days (adjusted $\mathrm{HR}=$ $0.67 ; 95 \%$ CI $0.48-0.95 ; p=0.003$ ) [16]. In addition, similar survival protection was seen in patients on betablockers who suffered a cerebrovascular insult (adjusted $\mathrm{HR}=0.03 ; 95 \%$ CI $0.002-0.54, p=0.017)$. It is possible that this observation may partly be explained by the immunomodulatory ability of beta-blockade. Beta-blockade has been associated with down-regulation of inflammatory responses, leading to improved penumbra conditions and thereby reduced secondary brain injury following CVI [14, 15]. Previous research in trauma has reported improved survival in patients on pre-injury betablockers suffering traumatic brain injury [17, 18]. Experimental studies have shown better cerebral perfusion and 
Table 4 Effects of beta-blocker exposure on the incidence of 1-year mortality in patients who suffered major postoperative complications

\begin{tabular}{lllr}
\hline Complication & $\begin{array}{l}\text { Adjusted HR } \\
\text { of BB }\end{array}$ & $95 \%$ CI & \multicolumn{1}{l}{$p$} \\
\hline Any major complication & 0.41 & $0.27-0.63$ & $<0.001$ \\
Sepsis & 0.10 & $0.03-0.38$ & 0.001 \\
Pneumonia* & 0.68 & $0.18-2.48$ & 0.554 \\
Anastomotic dehiscence & 0.25 & $0.03-2.18$ & 0.209 \\
DVT** $^{*}$ & 0.00 & $0.00-0.00$ & 1.000 \\
CVI* & 0.04 & $0.00-0.55$ & 0.017 \\
Wound infection* & 0.31 & $0.06-1.58$ & 0.159 \\
Intra-abdominal abscess* & 0.80 & $0.30-2.13$ & 0.650 \\
Bleeding* & 0.97 & $0.08-11.63$ & 0.977 \\
Stoma-related** & 0.00 & $0.00-0.00$ & 1.000 \\
Cardiovascular* & 0.33 & $0.18-0.64$ & $<0.001$ \\
Other** & 0.45 & $0.06-3.58$ & 0.449 \\
\hline
\end{tabular}

Proportional hazards Cox regression model was used, adjusting for sex, age, BMI, ASA, surgical procedure, indication for surgery, TNM classification and overall cancer stage

$H R$ hazard ratio, $B B$ beta-blockers, $C I$ confidence interval, $D V T$ deep vein thrombosis, $C V I$ cerebrovascular insult

*Adjusted for sex, age, BMI

**No adjustment

oxygenation in traumatic brain injury animal models, which in part, could explain these positive findings [19].

The increased risk of cardiovascular insults in the periand postoperative period following surgery has been known for long [8]. The major impact of cardiac complications on long-term survival was outlined by Khuri et al. who showed an increase in 1-year mortality following colectomy from 17.5 to $92.4 \%$ and 18.6 to $58.2 \%$ as a consequence of suffering a cardiac arrest or myocardial infarction, respectively [2]. Protective effects from betablockade on postoperative cardiac deaths have been seen in patients undergoing non-cardiac elective surgery [20]. Specifically, a sixfold increase in adverse events following non-cardiac surgery has been seen in patients with an increased cardiac risk profile who fail to continue their regular beta-blocker treatment [21]. In the current study, preoperative beta-blocker use did not reduce the incidence of major cardiovascular events following emergency surgery (adjusted IRR $=0.92 ; 95 \%$ CI $0.58-1.46 ; p=0.75$ ). However, patients who suffered a severe cardiovascular complication demonstrated improved 1-year survival in the presence of beta-blocker therapy (adjusted $\mathrm{HR}=0.33$; 95\% CI $0.17-0.63, p<0.001)$. In contrast to the current study, the well-known randomised controlled trial (POISE) detected a decrease in adverse cardiovascular outcomes but a higher risk of mortality in patients receiving preoperative beta-blockers [22]. There are several differences between these studies that should be emphasized when comparing results. In the current study, only severe cardiovascular events, classified using Clavien-Dindo, were included. This results in a lower number of adverse events that could lead to a statistical type two error. Further, in contrast to the POISE trial, restricted inclusion of patients who were subjected to emergency abdominal surgery for one surgical disease only do offer a more homogenous study population. The POISE trial focused on non-cardiac surgery and consequently included orthopedic, vascular and intra-abdominal surgery without distinction between surgical procedure performed, surgical approach (minimal invasive or open surgery) and urgency of the procedure. This results in a more heterogenous study population in terms of risk for postoperative complications and mortality. Finally, the POISE trial detected more cerebral events in the betablocked cohort. There was no increased risk for such adverse outcome in the current study which is in line with other more recent trials [23]. One possible explanation for this could be due to dosage differences. The methodology of the POISE trial involved the administration of $200 \mathrm{mg}$ extended-release metoprolol given preoperatively to betablocker naïve patients, whereas in the current study patients were exposed to lower doses of beta-blocker during a longer period of time prior to their surgery.

The current study outlines the impact of major postoperative complications on long-term survival following emergency surgery for colon cancer and how the outcome following some major complications may be improved by the exposure to a beta-blocker agent. The study is strengthened by its use of an externally validated prospective national patient registry with a coverage of over 99\% of all colon cancer patients in Sweden as well as by the all-encompassing national drug registry. The retrospective design of the study does, however, result in certain limitations. This includes the lack of co-morbidity data and detailed information around anesthesia and perioperative care, as well as the specific postoperative complications suffered and the intervention undertaken as result of these adverse events. The authors are also unable to account for the indication for beta-blockers being prescribed and the exact timing of administration for patients' regular betablocker agents whilst in hospital. Only patients with betablocker prescriptions that were collected were considered beta-blocker exposed; however, we are unable to control for a strict day to day compliance and the continued administration during the hospital stay and after hospital discharge. According to national guidelines set by the Swedish Society of Anesthesiology and Intensive Care, supported internationally by the guidelines set by the American College of Cardiology and American Heart Association (ACC/AHA), a patient on daily beta-blocker therapy should not be discontinued on their beta-blocker agent before or after undergoing non-cardiac surgery. It is 
therefore an assumption, backed up by national and international guidelines, that patients who in this study are labelled beta-blocker positive preoperatively continued to receive their beta-blocker both in hospital and following discharge. Further, due to the retrospective nature, we are also unable to account for the exact experience of the operating surgeon and the course of surgery. A prospective interventional study is required to address these issues and to validate current findings.

The development of major postoperative complications after emergency surgery for colon cancer is associated with an increased mortality risk during the first postoperative year. This finding advocates for measures taken to reduce such deleterious events but might also justify extended follow-up after discharge and timely interventions to minimize the risk of premature deaths. Beta-blocker therapy may provide a protective effect in these instances; however, this requires further research before any therapeutic recommendations for colon cancer patients could be issued.

\section{Compliance with ethical standards}

Conflict of interest All authors declare that they have no conflict of interest.

Open Access This article is distributed under the terms of the Creative Commons Attribution 4.0 International License (http://crea tivecommons.org/licenses/by/4.0/), which permits unrestricted use, distribution, and reproduction in any medium, provided you give appropriate credit to the original author(s) and the source, provide a link to the Creative Commons license, and indicate if changes were made.

\section{References}

1. Moonesinghe SR, Harris S, Mythen MG, Rowan KM, Haddad FS, Emberton M et al (2014) Survival after postoperative morbidity: a longitudinal observational cohort study. $\mathrm{Br} \mathrm{J}$ Anaesth 113(6):977-984

2. Khuri SF, Henderson WG, DePalma RG, Mosca C, Healey NA, Kumbhani DJ et al (2005) Determinants of long-term survival after major surgery and the adverse effect of postoperative complications. Ann Surg 242(3):326-341 (discussion 341)

3. Clarke A, Murdoch H, Thomas MJ, Cook TM, Peden CJ (2011) Mortality and postoperative care after emergency laparotomy. Eur J Anaesthesiol 28(1):16-19

4. Cohen ME, Bilimoria KY, Ko CY, Hall BL (2009) Development of an American College of Surgeons National Surgery Quality Improvement Program: morbidity and mortality risk calculator for colorectal surgery. J Am Coll Surg 208(6):1009-1016

5. Sjo OH, Larsen S, Lunde OC, Nesbakken A (2009) Short term outcome after emergency and elective surgery for colon cancer. Colorectal Dis 11(7):733-739

6. Ahl R, Matthiessen P, Fang X, Cao Y, Sjolin G, Lindgren R et al (2018) Effect of beta-blocker therapy on early mortality after emergency colonic cancer surgery. Br J Surg 106(4):477-483
7. Ahl R, Matthiessen P, Fang X, Cao Y, Sjolin G, Lindgren R, et al (2018) $\beta$-blockade in rectal cancer surgery: a simple measure of improving outcomes. Ann Surg. https://doi.org/10.1097/SLA. 0000000000002970

8. Desborough JP (2000) The stress response to trauma and surgery. Br J Anaesth 85(1):109-117

9. Swedish Colorectal Cancer Registry (SCRCR) [Internet]. http:// kvalitetsregister.se/englishpages/findaregistry/registerarkivengl ish/swedishcolorectalcancerregistryscrcr.2156.htm. Accessed 24 Feb 2018

10. White IR, Royston P, Wood AM (2011) Multiple imputation using chained equations: issues and guidance for practice. Stat Med 30(4):377-399

11. Rubin DB (2004) Multiple imputation for nonresponse in surveys, vol 81. Wiley, New York

12. Jestin P, Nilsson J, Heurgren M, Pahlman L, Glimelius B, Gunnarsson U (2005) Emergency surgery for colonic cancer in a defined population. Br J Surg 92(1):94-100

13. Banz VM, Jakob SM, Inderbitzin D (2011) Review article: improving outcome after major surgery: pathophysiological considerations. Anesth Analg 112(5):1147-1155

14. Rough J, Engdahl R, Opperman K, Yerrum S, Monroy MA, Daly JM (2009) beta2 Adrenoreceptor blockade attenuates the hyperinflammatory response induced by traumatic injury. Surgery 145(2):235-242

15. Friese RS, Barber R, McBride D, Bender J, Gentilello LM (2008) Could Beta blockade improve outcome after injury by modulating inflammatory profiles? J Trauma 64(4):1061-1068

16. Fuchs C, Wauschkuhn S, Scheer C, Vollmer M, Meissner K, Kuhn SO et al (2017) Continuing chronic beta-blockade in the acute phase of severe sepsis and septic shock is associated with decreased mortality rates up to 90 days. $\mathrm{Br} \mathrm{J}$ Anaesth 119(4):616-625

17. Mohseni S, Talving P, Wallin G, Ljungqvist O, Riddez L (2014) Preinjury beta-blockade is protective in isolated severe traumatic brain injury. J Trauma Acute Care Surg 76(3):804-808

18. Mohseni S, Talving P, Thelin EP, Wallin G, Ljungqvist O, Riddez L (2015) The effect of beta-blockade on survival after isolated severe traumatic brain injury. World $J$ Surg 39(8):2076-2083. https://doi.org/10.1007/s00268-015-3039-z

19. Ley EJ, Scehnet J, Park R, Schroff S, Dagliyan G, Conti PS et al (2009) The in vivo effect of propranolol on cerebral perfusion and hypoxia after traumatic brain injury. J Trauma 66(1):154-159 (discussion 159)

20. Lindenauer PK, Pekow P, Wang K, Mamidi DK, Gutierrez B, Benjamin EM (2005) Perioperative beta-blocker therapy and mortality after major noncardiac surgery. $N$ Engl $J$ Med 353(4):349-361

21. Kwon S, Thompson R, Florence M, Maier R, McIntyre L, Rogers $T$ et al (2012) Beta-blocker continuation after noncardiac surgery: a report from the surgical care and outcomes assessment program. Arch Surg 147(5):467-473

22. Devereaux PJ, Yang H, Yusuf S, Guyatt G, Leslie K, Villar JC et al (2008) Effects of extended-release metoprolol succinate in patients undergoing non-cardiac surgery (POISE trial): a randomised controlled trial. Lancet 371(9627):1839-1847

23. Obeid T, Arhuidese I, Gaidry A, Qazi U, Abularrage C, Goodney $\mathrm{P}$ et al (2016) Beta-blocker use is associated with lower stroke and death after carotid artery stenting. J Vasc Surg 63(2):363-369

Publisher's Note Springer Nature remains neutral with regard to jurisdictional claims in published maps and institutional affiliations. 